\title{
A Concept of Replicated Remote Method Invocation*
}

\author{
Jerzy Brzezinski and Cezary Sobaniec \\ Institute of Computing Science \\ Poznan University of Technology, Poland \\ \{Jerzy.Brzezinski, Cezary.Sobaniec\}@cs.put.poznan.pl
}

\begin{abstract}
In this paper we present a new infrastructure for building distributed applications that communicate through remote objects. The objects are available by the use of the Java RMI, enhanced by replication. Shared objects may be arbitrary complex with references to other objects allowing nested invocations. The replication process may be controlled and tuned by careful design of shared objects granularity, explicit synchronization operations, and replication domains definitions.
\end{abstract}

\section{Introduction}

Replication is one of the key concepts in distributed systems, introduced mainly for increasing data availability and performance. It consists in maintaining multiple copies of data items (objects) on different servers. However, replication introduces some serious problems like consistency. Centralized systems have only one version of every object, and its state at any time reflects the last write operation on that object. In a distributed system the notion of last is not so obvious because of the lack of a common clock. Every system using replication must therefore use some kind of consistency protocol that will arrange communication between replicating servers. Consistency protocol is a practical implementation of a consistency model that defines guarantees provided by the system regarding operation execution order.

In this paper we present a concept of a system for sharing objects in a distributed environment that supports replication. The fact of replica existence must be known by the user, and the system expects some cooperation in order to maintain the replicas in consistent state. We use Java RMI as the basic operation model, because it is a widely accepted solution, and allows tight integration of remote objects with application code.

Java Remote Method Invocation [1] is an extension of the Java programming language and its Java Object Model into Distributed Object Model [2]. It allows applications to call methods of remote objects in the same manner as they call methods of local objects. Essentially it is a kind of remote procedure call known

\footnotetext{
* This work was supported in part by the State Committee for Scientific Research
} (KBN), Poland, under grant KBN 7 T11C 03621 
from such products as Sun RPC. The unique feature of RMI is that it seamlessly integrates with Java programming language hiding the fact of true object location. On the client side the application uses an automatically generated $s t u b$ that implements the interface of the shared object. The stub intercepts all method invocations, transmits the invocation with arguments to the server side, receives the results, and finally returns them to the application. On the server side there is a skeleton that receives a method invocation from a client, unpacks it, and issues an appropriate method of a locally available object.

Related work. CORBA and DCOM are example technology standards that define a distributed object model which allows invocations on remote objects in a very similar way local methods are invoked. Because of their emphasize on interoperability they are not so tightly integrated with a programming language as the Java RMI. What is more important: they do not support replication.

NinjaRMI [3] is a ground-up reimplementation of Java RMI with three important enhancements: UDP unicast, UDP multicast, and cryptography support for reliable, unicast RMI. The system optimizes communication, and is a good base to build a replication system on top of it.

JGroup [4] uses a group communication approach. A specialized group reference maintains references to all replicas and uses a reliable unicast when communicating replicas. Clients invoke methods of the group reference which causes an iteration process through replicas until a working one is found. JGroup increases reliability but does not solve the problem of consistency.

Manta [5] generalizes standard RMI by providing a Group Method Invocation. Methods can be invoked on a local copy, on a remote object, or on an explicit group of objects. GMI may be therefore treated as an implementation tool for building a replication system.

Aroma [6] intercepts remote method calls and propagates them to other replicas. Method invocations are transfered using underlying group communication with reliable, totally ordered multicast protocol to provide strong replica consistency. One of the most important drawbacks of this approach is a consequence of the most important advantage: full transparency. Intercepting, decoding, and distributing method invocations is a costly process.

Highly efficient replication was used for a long time in Distributed Shared Memory (DSM) systems. They usually relax memory consistency model in order to achieve a better performance. However, DSM systems are usually memoryoriented instead of object-oriented. Objects in DSM systems are usually simple encapsulations of data, and do not allow to build complex object structures.

Replicated Remote Method Invocation (RRMI) is an efficient object replication infrastructure that will increase the availability of shared remote objects. The system seamlessly integrates with the Java object model thanks to the RMI concept, and, on the other hand, provides efficient replication using consistency protocols adapted from DSM systems. The shared object model does not restrict possible complexity of object structures, allowing the developer to create objects that reference other objects, with methods that call methods of other objects. 
We give the developer tools for optimizing the replication management such as: explicit synchronization and replication domains.

\section{Design of RRMI}

Replication strategy. Object replication in general is a complex task resulting from potential object complexity. Our previous experience 7 concerning object replication was limited to simple read-write objects, where objects may be treated as variables wrapped with objects that provide simple access methods for these variables. General objects provide methods that modify their state in an arbitrary way. The first problem that arises while replicating such objects is distinction between methods: which methods modify the state, and which are read-only methods. The problem may be generalized to the problem of identification of commuting methods, i.e. methods that may be safely executed concurrently, possibly on different replicas. A simple example is a queue object that can execute concurrently two modifying methods add and get unless it is empty. In RRMI the user annotates the shared object interface with statements indicating whether the method is a modifying one or not. The statements are then interpreted by a stub compiler to optimize method invocations.

Replication requires some kind of consistency management in order to hide replicas divergence from the user. An important part of consistency management is updating policy. Objects can be updated by means of state transfer or operation transfer. State transfer is usually used by DSM systems which use a flat page of memory as the basic sharing structure. In such systems update consists in transferring the whole updated page, or in computing so called diffs [8] that efficiently represent changes applied to memory page. Tracking changes made on objects, and especially object structures, is not that simple, and causes considerable computing overhead. The alternative is to transfer the whole object state every time it changes, which results in unacceptable communication overhead. For these reasons we have chosen operation transfer as the basic replica update policy.

Usually replication techniques are divided into two distinctive groups: passive replication and active replication [9]. The classification is somehow related to replica update policy but it emphasizes another aspect of replication: the organization aspect. In passive replication the processing takes place only at a single server, called the primary replica, and then is transfered (using state transfer) to other replicas. In active replication all replicas are contacted directly by the client, and are instructed to execute a given processing. Our approach is a mixture of these two approaches. Clients of RRMI contact only a single server (as in passive replication), the server registers appropriate method invocations on shared objects, and later sends the invocation history to other replicas where the methods are re-executed. This procedure assumes obviously a deterministic processing on replicas (as in active replication). Our system tries to be as lazy as possible: updates are sent to the nodes that really need them, usually after explicit requests. 
State transfer systems are easy to implement because they require keeping only the newest version of all objects in the system with some kind of timestamps indicating its version. When a replica needs to be updated, the current version is located, transfered, and replaces the old one, regardless of the state of the replica. This is not the case in advanced state transfer systems (i.e. using diffs), nor operation transfer systems. They need to apply all changes made on an object by all servers, and apply them in order. In case of object replication system that permits references between objects and therefore nested calls, it is also necessary to apply the changes in causal order. We register and reconstruct the causal order of method invocations by the use of vector clocks which are also used by the consistency manager (see Sect. 3).

Shared object structure. Objects are very different entities. They may be as small as single variables, or as large as databases. This raises important problems when designing a system that provides replication facility of such objects. Every object registered and maintained by the replication infrastructure needs some additional data structures that keep track of its replicas consistency. The best performance can be achieved when the size of maintenance structures is small compared to the size of shared objects. The size of a shared object therefore should be as large as possible. However, this limits the possible concurrency level of applications accessing shared objects. In an extreme case the system would handle a single shared object with permanent access conflicts on replicas that are constantly inconsistent. In order to minimize possible number of conflicts and to increase concurrency, the shared data space should be divided into small, possibly autonomous entities. This claim is however in conflict with the previous one. In RRMI we leave the decision of object granularity to the user. He is responsible for designing shared objects in a manner that will be the best possible tradeoff between concurrency and maintenance overhead. The system does not have enough knowledge about the application characteristics to make the decision in a better way.

Construction of more advanced objects very often requires the use of other objects because they are inherently complex. For example, one object may consist of a couple of other objects. Our system does not require explicit registration of all internal objects within the shared space. It is only necessary to register the top-level, interface objects. The whole internal structure will be replicated along with the main object. This concept is similar to clouds of objects used in Manta [5]. However, our objects may still have references to other shared objects which is not the case in Manta clouds. We use regular Java references to point to internal objects, and remote references to point to other shared objects. The kind of reference therefore semantically defines boundaries of replication entities.

Nested invocations. Objects are not isolated structures. Usually they are in relations with other objects which is expressed in programming languages as references to other objects. References allow invocations of other objects' methods, that is nested invocations. Pure active replication systems that permit nested invocations face the problem of repeated calls when the nested object is called 
several times by its callee that is also replicated. The system should either detect such repeated calls and silently ignore them, or avoid them by ignoring some calls at their source. RRMI solve the problem in a slightly different manner. Because of the replication strategy, the objects are called directly by the client only once. Such calls are then transmitted to replicas, but the re-execution is explicit, therefore may be treated differently — it is just not replicated. The only method invocations that are replicated come directly from the user. Whenever a nested method is called by a shared object, it is executed only on locally available replica of that object. Such nested calls are not registered in the history, and are not replicated. This assumes that every shared object reachable through remote references is available locally.

Naming. In standard RMI, objects, before they can be accessed, are bound to a name within a registry. Registry is a simple naming service that holds an array of objects' stubs, and sends them to clients when they issue lookup operation. The stub internally contains enough information to locate the server that provides the object implementation, i.e. its address and port number. The standard implementation does not assume replication, so every object is registered only once, at the server that provides its implementation. In an environment supporting replication the registry is aware of object replicas and returns stubs pointing to the nearest server. "Near" may be defined differently: based on the network delay, latencies, server load, and other factors.

The process of object creation and registration needs to be done atomically in order to avoid conflicting name resolutions at different servers. These operations are integrated within the consistency management. Successful object creation guarantees that there was no other object with a given name before that creation.

Remote references may be passed by value between shared objects as method parameters. When such a reference is transfered to another server, its representation is updated to point to that server, if the server holds a replica of that object. This is necessary to properly handle nested calls: it was assumed that replicas of objects that are referenced are available locally, at the same server.

Replication domains. Objects in RRMI are allowed to call methods of other objects because they are expected to be available locally. In the worst case, when there is some kind of link between every object in the system, the assumption will require total replication of all objects. Obviously this does not lead to optimal efficiency, because some replicas will be updated even when they are not accessed by anyone. We introduce a new concept of replication domains [10 in order to alleviate the problem. A replication domain essentially is a set of shared objects that may reference each other, and a set of servers that maintain their replicas. There are no references between objects from different domains. Objects from different domains may be replicated on a different set of servers. The concept reflects different requirements of concurrent applications that run on top of RRMI. Applications have different expectations concerning objects availability, and their consistency management. The programmer is responsible for defining replication domains and objects' associations to domains. 
Transport layer. One of the most important factors that influence the availability of remote objects is the communication infrastructure that is used to access the objects or their replicas. We use two different transport mechanisms to communicate between processes. Remote invocations coming from clients are transfered using TCP connections. However, in contrary to the standard RMI implementation, we establish a single connection between a client and a server, and keep it open between consecutive calls. Java RMI uses a new TCP connection every time a method is called, which may result in a poor efficiency if the methods are issued frequently. On the other hand, a client is usually located at the same host as the server being contacted by the client, or on a host that is located close to such a server (e.g. within the same LAN). As a consequence, the connection overhead introduced by TCP protocol remains low, and its reliability gives the client precise monitoring of the link status.

Communication between servers maintaining objects' replicas is carried out differently. The servers are located on different nodes, usually distant. In order to facilitate and optimize the communication we have developed a new message passing library dedicated for the Java environment. It is called JMPL - Java Message Passing Library. The library was used in our previous project Jash [7. RRMI uses the second revision of JMPL which is substantially rewritten and enhanced. Currently the library is much more flexible, supports dynamic reconfiguration, uses new features of the Java language, and is ready to work in an unreliable environment. JMPL uses UDP as the main transport protocol, but provides typical features of the TCP protocol like: reliable communication, ordering, flow control, message fragmentation, or windowing.

\section{Consistency Maintenance}

Replication introduces the problem of consistency when replicas are allowed to change. The problem becomes more complicated when modifications can be done concurrently at different replicas, i.e. in a multi-master model. Concurrent conflicting writes should not be allowed, or, in an optimistic approach [11], detected and resolved.

Our approach in RRMI system at this stage of development is close to pessimistic replication. It means that it is not possible to submit concurrent, conflicting object updates. Such updates would require a roll-back mechanism that is necessary to reschedule non-commutative operations in the case of a conflict. However, RRMI tries to postpone update propagations to maximum extent in order to minimize delays when submitting modifications to a shared object.

Traditionally, one of the most important goals of systems supporting replication in a distributed system was to hide the fact of replication from the user, i.e. to make the replication as transparent as possible. Although possible, this transparency comes at a cost. Usually it requires a kind of strong consistency where all replicas are updated or invalidated at the same time. Such protocols tend to be highly inefficient, and are only useful in systems with a high read/write operation ratio. Other models have been developed and they relax 
somehow consistency constrains at the cost of user awareness of the replication. In RRMI we have used entry consistency (EC) [12] as the basic consistency model. Entry consistency is a kind of relaxed consistency that uses special synchronization operations on locks. These operations are used explicitly by the user to instruct the underlying replication managers when and where a given portion of data should be updated. There are two synchronization operations: acquire and release, that indicate blocks of code when a replica should be updated. EC additionally distinguishes between acquires in shared mode and acquires in exclusive mode, in order to make possible concurrent reads of objects. Another unique feature of EC compared to release consistency $(\mathrm{RC})$, is that it requires explicit association of shared objects to locks. In RC the association is implicit and may be deduced by analyzing the code. RRMI is a public system that is designed to be used by different applications at the same time. For this reason, the associations of locks to shared objects should be explicit, and available for applications communicating only with the system.

The consistency management layer intercepts method invocations in stubs, especially in server stubs. The user is expected to select a lock for every shared object during object creation. Several objects may be synchronized using a single lock. Consider the following example code:

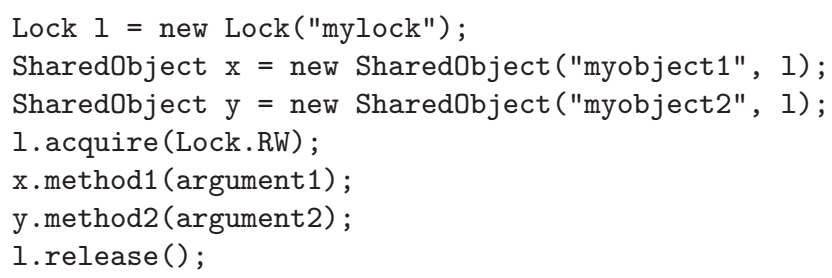

Here, the lock was explicitly acquired. The same processing may be also done using implicit synchronization. The server stub will detect that the appropriate lock has not been acquired, and will acquire it before calling the method, and release after the method returns. This model is close to strong consistency, and is attractive for its full transparency. However, it does not allow the user to optimize the processing in order to decrease the number of synchronization operations. In the example, there is no synchronization operations between method1() and method2(), neither explicit nor implicit. Explicit synchronization may be necessary in deadlock prevention. Another possible usage of this technique is an atomic update of a set of objects, an operation not possible with strong consistency without implementing additional synchronization mechanisms.

Processing history. Modifications of objects are not sent directly to other servers right after their submission. RRMI uses operation transfer which requires gathering information about the processing. This is done by maintaining histories of processing at every server. Servers register all top level local method invocations submitted by clients, and incorporate parts of histories from other servers sent during synchronization operations (namely acquires). The histories are applied at servers in causal order. Causal order is preserved thanks to vector clocks that represent number of synchronization operations (acquires) issued at servers. 


\section{Conclusions}

In this paper we have presented a new infrastructure for building distributed applications that communicate through remote objects. The construction of the system is currently being finalized, and we expect to get more practical experience and carry out some performance evaluation tests. Future work will be concentrated on introducing other consistency models, especially those which are more client-oriented, and on increasing dependability of the system through object recovery in case of server crashes.

\section{References}

1. Sun Microsystems: Java Remote Method Invocation Specification. (1998)

2. Wollrath, A., Riggs, R., Waldo, J.: A distributed object model for the Java system. In: 2nd Conference on Object-Oriented Technologies \& Systems (COOTS), USENIX Association (1996) 219-232

3. Gribble, S.D., Welsh, M., Brewer, E.A., Culler, D.: The MultiSpace: An evolutionary platform for infrastructural services. In: In Proc. of the 16th USENIX Annual Technical Conference, Monterey, CA, USA (1999) 157-170

4. Montresor, A.: The JGroup distributed object model. In: Proc. of the Intl. Working Conference on Distributed Applications and Interoperable Systems, Helsinki, Finland (1999) 389-402

5. Maassen, J., Kielmann, T., Bal, H.E.: Parallel application experience with replicated method invocation. Accepted for publication in Concurrency and Computation: Practice and Experience (2001)

6. Narasimhan, N., Moser, L.E., Melliar-Smith, P.M.: Transparent consistent replication of Java RMI objects. In: Proc. of the International Symposium on Distributed Objects and Applications (DOA'00), Antwerp, Belgium (2000)

7. Brzeziñski, J., Sobaniec, C., Szychowiak, M., Wawrzyniak, D.: Jash: A platform for sharing data in distributed Java applications. In: Proc. of the Int'l Conf. on Parallel and Distributed Processing Techniques and Applications (PDPTA '98), Las Vegas, USA (1998) 1430-1437

8. Amza, C., Cox, A.L., Dwarkadas, S., Keleher, P., Lu, H., Rajamony, R., Yu, W., Zwaenepoel, W.: Treadmarks: Shared memory computing on networks of workstations. IEEE Computer 29 (1996) 18-28

9. Pedone, F., Wiesmann, M., Schiper, A., Kemme, B., Alonso, G.: Understanding replication in databases and distributed systems. In: Proc. of the 20th International Conference on Distributed Computing Systems (ICDCS'00), Taipei, Taiwan (2000) 464-474

10. Sobaniec, C., Wawrzyniak, D.: Using domains to support replication in a distributed shared object system. In: Proc. of Int'l Conf. on Parallel and Distributed Computing and Networks (PDCN 2004), Innsbruck, Austria (2004)

11. Saito, Y., Shapiro, M.: Optimistic replication. Technical Report MSR-TR-2003-60, Microsoft Research (2003)

12. Bershad, B.N., Zekauskas, M.J., Sawdon, W.A.: The Midway distributed shared memory system. In: Proc. of the 38th IEEE Int'l Computer Conf. (COMPCON Spring'93). (1993) 528-537 